\title{
Internal thermal amplification for increasing the responsivity of pyroelectric detectors
}

\author{
Querner, Yvonne \\ Technische Universität Dresden \\ Solid-State Electronics Laboratory \\ 01062 Dresden \\ Germany
}

\begin{abstract}
A novel approach for increasing the sensitivity of pyroelectric detectors and its mathematical and physical analysis are presented in this paper. Due to a three-dimensional pattern etched into the sensitive element lateral heat flux spreading is used to improve the responsivity. Here, the effect is applied, that very thin regions between thicker ones show a faster heating due to incident radiation and, hence, lead to an additional heat flow from these intermediate regions to the sensitive element. The analysis allows the description of the thermal and electrical behavior of the sensitive element depending on the dimensions of the pattern and the modulation frequency. A short overview about the technology of structured elements will be given.
\end{abstract}

Keywords: Pyroelectric detector; thermal simulation; patterned layers; high responsivity

\section{Introduction}

Pyroelectric detectors are widely used in many applications like pyrometry, security technology, gas analysis and spectrometry. For many of these applications a high responsivity and a high detectivity are required. In order to get maximum responsivity, several approaches were already investigated, for example the appropriate choice of pyroelectric material, special construction techniques to reduce the heat conduction away from the sensitive element or the use of thermal absorber layers [1, 2].

This paper studies another method for increasing responsivity and detectivity. It uses a threedimensionally structured thermal element having locally reduced passive areas and thicker electrically active areas. So far, only the reduction of the whole element thickness $d_{a}$ was considered that leads to a reduced heat capacity of the element and therefore to a higher temperature change due to the absorbed heat flow. But simultaneously, the electrical capacity of the element increases which again decreases responsivity. Therefore, the responsivity does not change at all in particular for modulation frequencies over $10 \mathrm{~Hz}$.

The new structure uses the advantages of the higher detectivity of thinner elements and the higher responsivity of thicker elements. First simulation and measurement results depending on design parameters will be presented.

\section{Basic Idea}

In pyroelectric detectors the pyroelectric effect is used to transform an incident heat flow into a temperature change and after a thermal-electrical conversion into a measureable voltage, see figure 1.

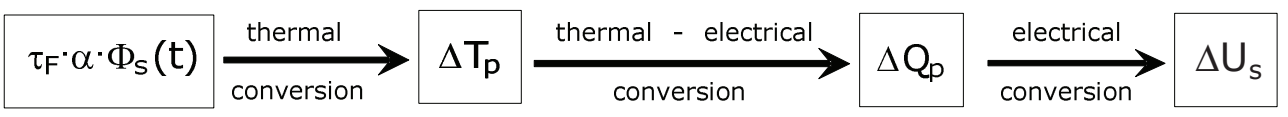

Fig.1. Schematical illustration of the pyroelectric effect. $\Phi_{s}$ incident radiation on sensor element, $\alpha$ absorption coefficient, $T_{F}$ transmission coefficient, $\Delta T_{p}$ temperature change, $\Delta Q_{p}$ polarization change and $\Delta U_{s}$ voltage change. 
In order to reach high responsivity and detectivity values such pyroelectric detectors usually use a sensitive element with a very thin but homogeneous thickness and perforation to reduce the heat flow away from the sensor [2].

This work studies a novel approach for increasing the responsivity of pyroelectric detectors in voltage mode by structuring the sensitive element with a three-dimensional pattern. The locally reduced thickness of the detector leads to a temperature gradient in the sensitive element which results in a heat flow from the passive to the active areas and causes a temperature rise in the active areas compared to nonstructured elements (Fig.2). Due to this effect the output signal of the sensor can be increased in contrast to unstructured sensors at the same incident radiation [3].

$\Phi(\mathrm{t})$

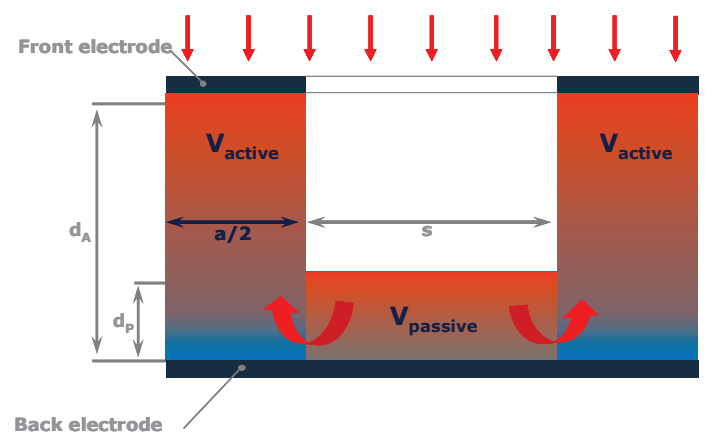

Fig.2. Cross-section of the structured sensitive element with electrically thicker active and thinner passive areas. The red arrows characterize the resultant heat flow from the passive to the active area. $s$ is the width of the passive regions.

\section{Modelling and Simulation}

\subsection{Thermal analysis - analytical and numerical simulations}

Due to the complex structure of the three-dimensionally patterned element the analytical calculations were performed by two simplified models. The first model neglects the spatial expansion of the element but takes the aligned material parameters into account resulting from the remaining pyroelectric material/removed material in the cavities ratio. The second model is based on the well-known partial differential equation for heat flow considering vertical and lateral heat conduction [2] which was adapted to the new structures by an average element thickness.

The finite element model was implemented in ANSYS ${ }^{\circledR}$ 11.0. A first finite element model was used to study the principle thermal procedures within the sensitive element. In order to keep the computing time justifiable, only a part of the three-dimensional pattern was simulated. Furthermore, the surrounding chip material and the sensor housing were neglected. The simulated element part is considered thermally isolated from the rest of the sensitive element. The incident radiation is modeled by a periodic heat flux at the surface of the element.

Figure $3 a$ shows the transition from the sensitive element to the FE-model. Figure $3 \mathrm{~b}$ presents the simulated temperature distribution in the active and passive area.

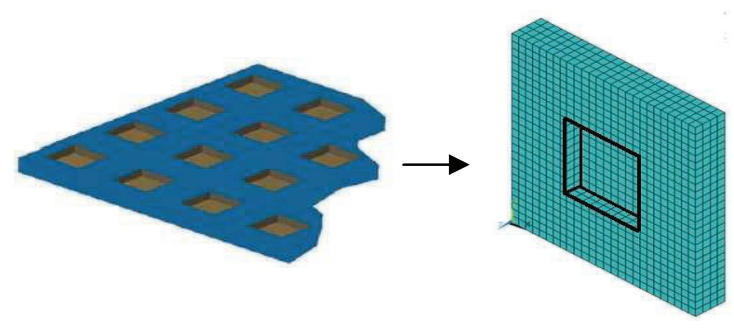

a)

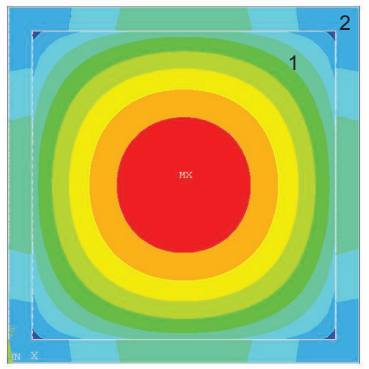

b)

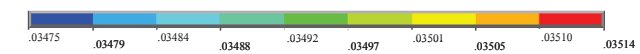

Fig.3. (a) Transition from the three-dimensionally structured sensitive element to the FE-model. (b) Temperature distribution in the active (2) and passive area (1). 
A second FE model considers the whole sensitive element with the etched structure in order to study the heat change with the surroundings. Radiation as well as convection heat were taken into account. The temperature distribution inside the sensitive element is shown in figure 4. Figure 5 presents the temperature amplitude (node temperature) versus time for different structure widths $s$. It could be shown, that the influence of the surrounding is of lower significance, whereas the geometry of the structure heavily influences the properties.

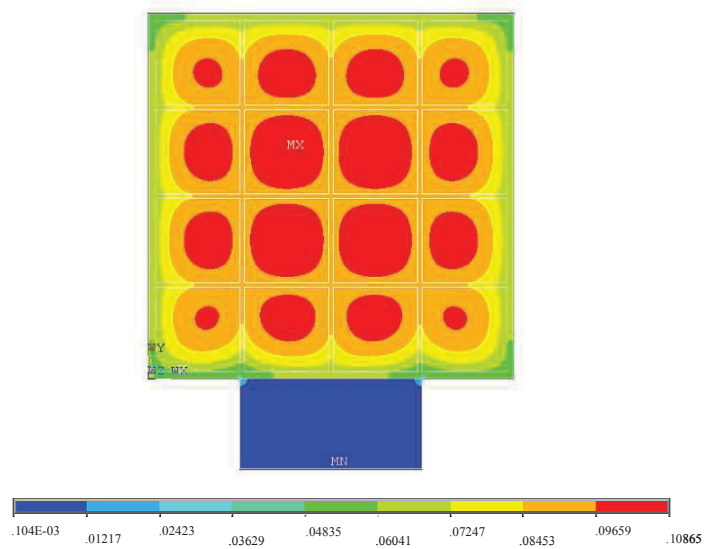

Fig.4. Finite element modeling of the temperature distribution in the whole sensitive element.

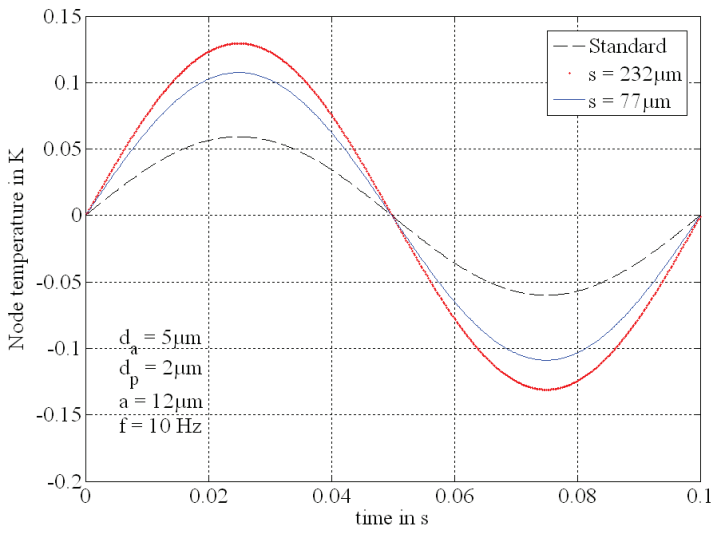

Fig.5. Node temperature of an unstructured sensor in comparison to different structure sizes $s$ at the same location (node).

\subsection{Electrical Analysis}

The three-dimensionally etched pattern in the sensitive element causes a change of the electrical capacity. Therefore, an electrical transfer function ETF was defined to describe the relation between the sensor voltage output $v_{s}$ and the temperature amplitude $T_{p}$ in the active volume of the sensitive element:

$$
E T F=\frac{v_{S}}{\hat{T}_{P}}=\frac{A_{F E} \cdot p \cdot \omega}{\sqrt{\left(\frac{1}{R}\right)^{2}+\omega^{2} \cdot C^{2}}}
$$

with:

$$
\begin{array}{ll}
A_{F E} & \text { sensitive area, } \\
p & \text { pyroelectric coefficient, } \\
\omega & \text { modulation frequency, } \\
R & \text { resistance of the sensor and } \\
C & \text { total capacity of the sensor. }
\end{array}
$$

$R$ is the electrical resistance of the whole sensor, including the electrical resistance of the sensitive element and the pre-amplifier. The capacity $C$ is the sum of the capacities of the pre-amplifier and the sensitive element. Due to the reduced sensitive area, the value of the electrical transfer function will always be less than that of unstructured sensors.

\section{Sensor fabrication}

The front electrode is evaporated onto the sensor, after a photo resist was deposited onto the substrate and patterned by lithography. Thereby, the grid structure is transferred into the resist with a pitch of $(s+$ a) in the region of the sensitive element. The rectangular structures are etched into the lithium tantalate substrate by ion beam milling. The depth of the structure depends on the etch time and amounts to about $5 \mu \mathrm{m}$. The substrate is thinned down to a thickness of $<20 \mu \mathrm{m}$ by lapping and polishing from the backside. In the area of the sensitive element the substrate is partially thinned down to about $6 \mu \mathrm{m}$ by ion beam etching. Therefore, the final thickness inside the structure amounts to about $1 \mu \mathrm{m}$. Finally, the back electrode is deposited by evaporation. Figures 6 and 7 show a confocal image of a part of the structured sensor and a REM image of the whole sensitive element, respectively. 


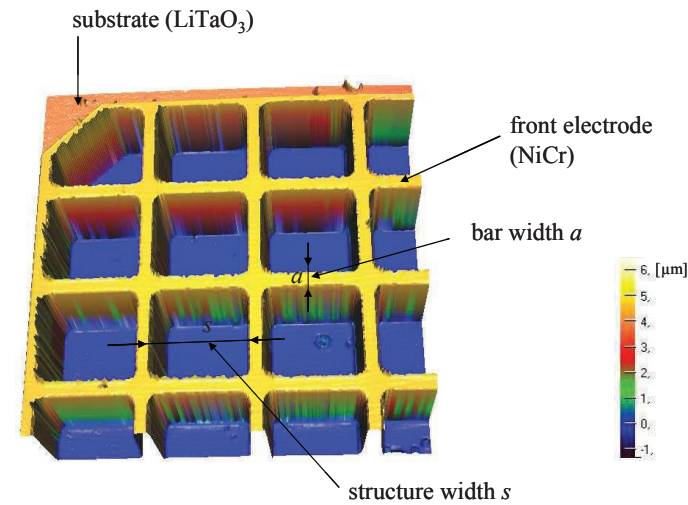

Fig.6. Confocal microscope image of a part of a structured sensitive element.

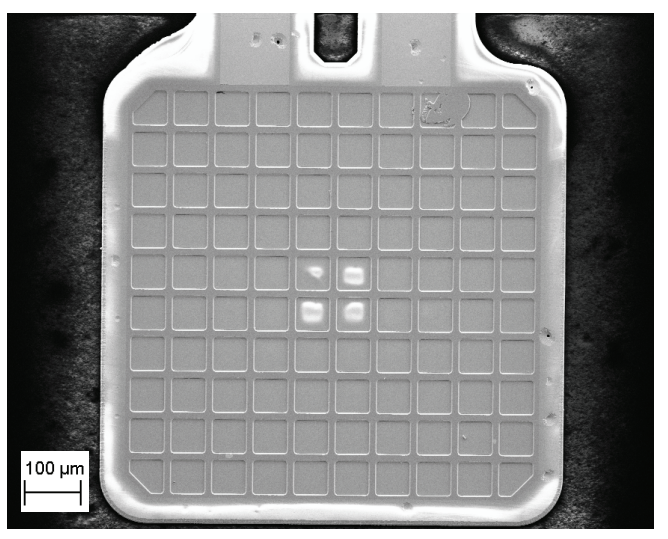

Fig.7. REM image of a structured element.

\section{Results and Discussion}

The thermal and electrical simulation are based on a single-element detector of lithium-tantalate $\left(\mathrm{LiTaO}_{3}\right)$ with an element thickness $d_{a}$ of $5 \mu \mathrm{m}$ and a sensitive element size of $1 \times 1 \mathrm{~mm}^{2}$. The etched cavities are filled with air.

The simulation results were used to calculate the responsivity $R$ and the electrical transfer function ETF of the structured sensors depending on the widths $s$ and $a$ of the passive and the the active areas, respectively.

\subsection{Thermal Simulation}

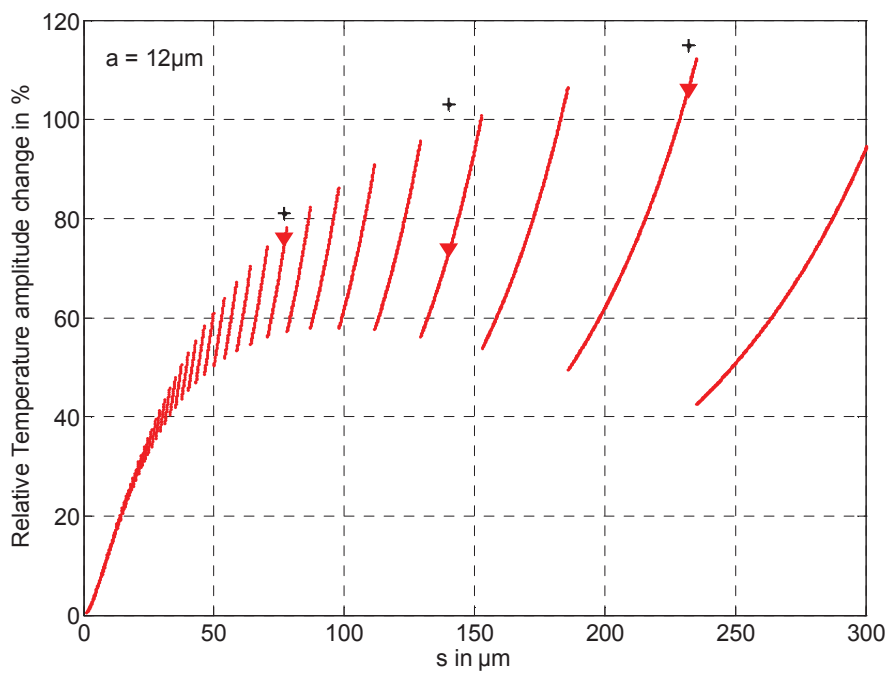

Fig.8. Relative temperature amplitude change depending on the structure width $s$; + FE results, $\boldsymbol{\nabla}$ analytical simulation results.

Figure 8 shows the comparison between the simulation results calculated analytically and numerically for different structure sizes $s$ and a thickness of $2 \mu \mathrm{m}$ of the passive areas. The discontinuities in the plot of the red line result from the integer number of the etched cavities in the element. As the curves in figure 8 show, the temperature amplitude can be increased up to $100 \%$ referred to an unstructured sensor.

\subsection{Electrical transfer function}

Figure 9 shows the change of the electrical transfer function ETF depending on the structure width $s$. It depends only on the size of the active area and the element thickness $d_{a}$, but is not influenced by the etch depth, because only the active region has an influence on the electrical capacity. 


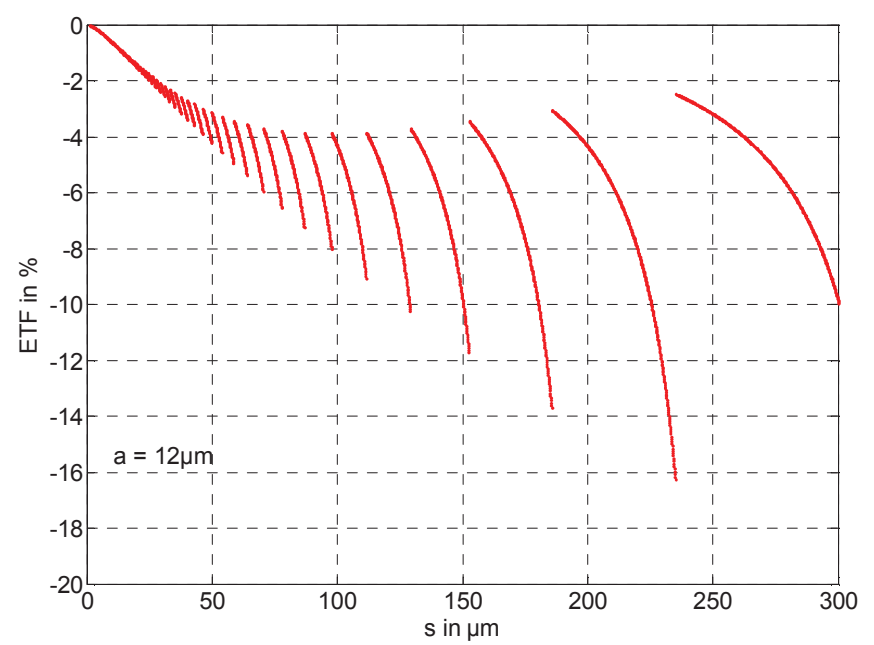

Fig. 9. Relative transfer function change $E T F(s) / E T F(0)$ depending on the structure size $s$, passive area thickness $d_{p}=2 \mu \mathrm{m}$.

\subsection{Responsivity}

Voltage responsivity of a pyroelectric detector element follows from electrical transfer function ETF (eq.(1)) and temperature amplitude $\hat{T}_{p}$ (figure 8):

$R_{v}=\frac{E T F \cdot \hat{T}_{p}}{\Phi}$.

Reduction in active sensor area leads to a decrease of the ETF and therefore to a smaller responsivity. Nevertheless, simulation results show, that the increase in temperature will always be larger than the reduction due to the lower electrical capacity for similar heat fluxes.

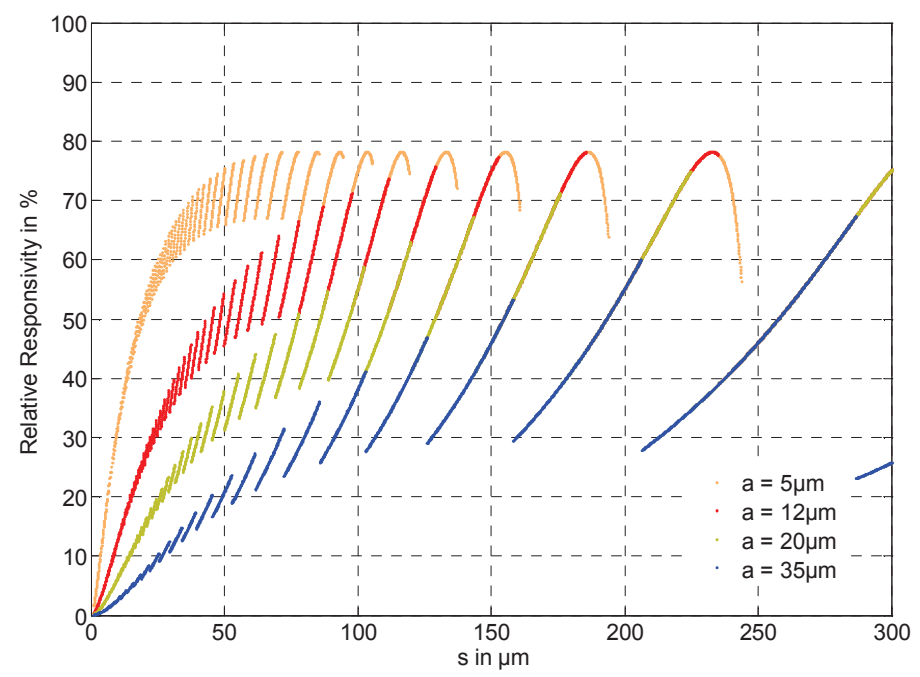

Fig.10. Relative responsivity change $R(s) / R(0)$ referred to an unstructured sensor depending on the structure size $s$ and the width $a$ of the active region.

Figure 10 shows that there is an optimum between element thickness $d_{a}$, passive area thickness $d_{p}$, active area width $a$ and structure width $s$. To achieve a high thermal amplification, an optimum combination of geometry parameters have to be chosen. For example, a sensor with an element 
thickness $d_{a}$ of $5 \mu \mathrm{m}$, a thickness $d_{p}$ of $2 \mu \mathrm{m}$, a structure width $s$ of $230 \mu \mathrm{m}$ and a width a of $12 \mu \mathrm{m}$, could lead to an increase in sensitivity of $80 \%$ compared to an unstructured sensor.

\subsection{Preliminary results}

First measurement results (see figure 11) show an increase of the voltage responsivity between 40 and $55 \%$. This values are still lower than the expected simulation results. One reason might be neglects in the rough model used here.

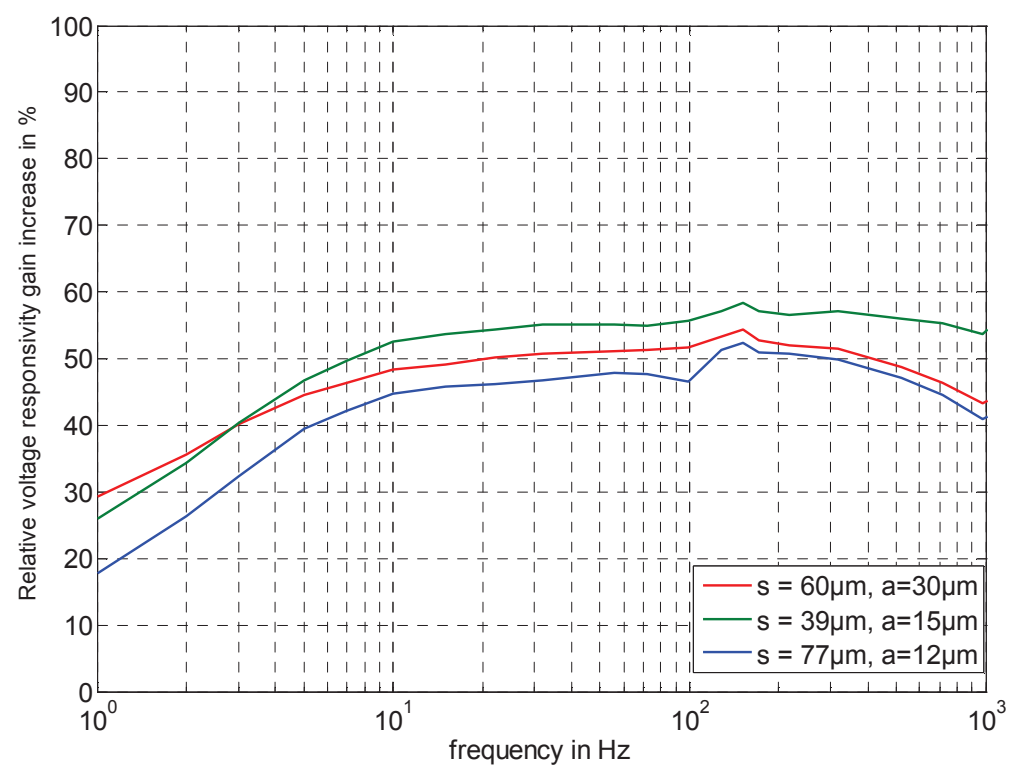

Fig.11. Relative responsivity gain $R(s) / R(0)$ related to an unstructured sensor depending on the modulation frequency and for different geometries.

\section{Conclusion}

It could be shown that three-dimensional patterning of the sensitive element of pyroelectric IR sensors has an positive influence on their sensor parameters. Depending on the structure width and other geometrical parameters, the achievable responsivity can be increased by 50 to $150 \%$ or even higher compared to unstructured detectors. Further studies will be performed to include the influence of absorber layers in the etched cavities.

\section{Acknowledgement}

The authors gratefully acknowledge financial support by the German Research Foundation (DFG). (Research grant GE 779/21-1).

\section{References}

[1] G. Milde et al., 3-D Modeling of Pyroelectric Sensor Arrays Part II: Modulation Transfer Function, IEEE Sensors Jounal, vol. 8, no. 12, Dec 2008

[2] S. Möhling, The influence of heat exchange between a sensitive element and its surroundings on the specific detectivity of pyroelectric detectors, Infrared Physics 34, no 5, 1993, S.487

[3] Patentschrift, DE 102009009342.7 